\title{
Optimal vibration control of beams with total and partial MR- fluid treatments
}

\author{
Vasudevan Rajamohan ${ }^{1}$, Ramin Sedaghati ${ }^{2}$, Subhash Rakheja ${ }^{2}$, \\ ${ }^{1}$ School of Mechanical and Building Sciences, \\ VIT University, Vellore, Tamilnadu, India, 632014. \\ ${ }^{2}$ CONCAVE Research centre, Department of Mechanical Engineering, \\ Concordia University, Montreal, Quebec, Canada, H3G1M8. \\ E-mail: vasudevan.r@vit.ac.in
}

\begin{abstract}
This study presents synthesis of full-state and limited state flexible mode shape (FMS)based controllers for suppression of free- and forced vibration of a cantilever beam fully and partially treated with the magneto-rheological (MR) fluid. The governing equations of motion of the three layer MR sandwich beam are expressed in the state variable form comprising a function of the control magnetic field. An optimal control strategy based on the linear quadratic regulator (LQR) and a full-state dynamic observer is formulated to suppress the vibration of the beam under a limited magnetic field intensity. The lower flexural mode shapes of the passive beam are used to obtain the estimates of the deflection states so as to formulate a limited state LQR control synthesis. The free-and forced vibration control performances of both the full-state observer-based and limited state FMS-based LQR control strategies are evaluated for the fully as well as partially treated MR-fluid sandwich beams. The results show that the full-state observer-based LQR control can substantially reduce the tip deflection responses and the settling time of the free vibration oscillations. The limited-state LQR control based on the mode shapes effectively adapts to the deflections of the closed-loop beams and thus yields vibration attenuation performance comparable to that of the full-state LQR controller. The partially-treated beam with MR-fluid concentration near the free end also yields vibration responses comparable to the fully treated beam, while the natural frequencies of the partially treated beams are considerably higher.
\end{abstract}

\section{Introduction}

Control of vibration in structures via active, semi-active and passive vibration isolation systems continues to be the focus of many studies. A wide range of active vibration control systems have shown significant performance gains, while their implementations have been mostly limited due to the high cost and power requirements [eg., He et al. 2001; Lam et al. 1997; Lee and Kim, 2001]. On the other hand, the passive systems are known to be most reliable, while fixed damping parameters involve a trade-off between the control of vibration at resonance and the higher frequency isolation performance [Harris, 1987, Wang and Wereley, 2002]. Alternatively, semi-active vibration control devices have shown to provide the fail-safe and reliable feature of the passive systems together with the performance gains comparable to those of the active devices with minimal power requirement [Nishitani and Inouve, 2001; Spencer Jr. and Nagarajah, 2003; Stanway et al., 1996; Ahn et al. 2005]. In particular, semi-active devices with smart fluids with controllable rheological properties such as Electrorheological (ER) and Magnetorheological (MR) fluids offer excellent potential for achieving control of vibration over a broad frequency range with only minimal external power [Choi et al, 2005; Liu et al. 2005]. Such fluids can provide significant and rapid 
changes in the damping and stiffness properties of structures with application of an electric or a magnetic field [Spencer Jr., et al., 1997; Carlson and Weiss, 1994; See, 2004]. Even though both the MR and ER fluids typically exhibit similar viscosity in their non-activated or "off" state, the MR fluids exhibit a much greater increase in viscosity when activated and need relatively low power compared to the ER fluids [See, 2004; Yao et al. 2002]. The yield stress of the MR fluid, MRF 100, is in the range of 2-3 kPa in the absence of a magnetic field, but it rapidly exceeds $80 \mathrm{kPa}$ under the application of a magnetic field in the order of 3000 Oe [Ahn et al., 2005].

The properties of ER and MR-fluid dampers have been widely characterized analytically and experimentally for vibration suppression of structures and systems [Pranoto et al., 2004; Dyke et al., 1998; Choi, 1999]. The reported structural models have generally employed lumped ER/MR dampers at selected discrete locations of the structures. Such models thus consider multiple damping elements to control the vibration corresponding to different modes, which would require complex controller designs. Alternatively, a few studies have applied ER/MR fluids in simple structure models to achieve controllable distributed properties by embedding ER/MR material layers between two elastic/metal layers. This approach can yield significant variations in distributed stiffness and damping properties of the structure, and thus offers superior potential for control of multiple vibration modes. Furthermore, the embedded MR/ER fluid treatments could yield more compact designs compared to the discrete damping treatments proposed in previous studies [Pranoto et al., 2004; Dyke et al., 1998; Choi, 1999]. While a number of studies have analyzed sandwich structures with ER fluids [Gandhi et al., 1989; Choi et al., 1990; Yalcintas and Coulter 1995; Yalcintas and Coulter 1998], the application of MR materials in sandwich structures have been explored in a very few studies over the past decade [Yalcintas and Dai, 1999; Yalcintas and Dai, 2004; Sun et al., 2003; Yeh and Shih, 2006].

Yalcintas and Dai [Yalcintas and Dai, 1999; Yalcintas and Dai, 2004] investigated the dynamic responses of a simply-supported sandwich beam comprising a MR fluid layer using the energy approach under transverse load. The study also compared the dynamic responses of the MR sandwich beam with those of the beam employing ER-fluid, and concluded that the MR fluid based adaptive structure can yield significantly higher natural frequencies, nearly twice that of the ER fluid based adaptive structure. Sun et al. (2003) also analyzed the dynamic responses of the MR sandwich beam experimentally and analytically using the energy approach, and derived relationships between the applied magnetic field and the complex shear modulus of the MR material using the oscillatory rheometry technique. The dynamic characteristics and instability of MR fluid treated cantilever structure subject to axial loading was investigated by Yeh and Shih (2006) using the DiTaranto (1965) sixth-order partial differential equation coupled with incremental harmonic balance method. Rajamohan et al. (2010a) derived finite-element and Ritz formulations for a sandwich beam with uniform MR-fluid treatment but various boundary conditions, and demonstrated their validity through experiments conducted on a cantilever sandwich beam. The study also proposed non-linear relationships between the complex shear moduli of the MR fluid and the applied magnetic field on the basis of the laboratory measured free vibration response.

The above studies have considered uniform MR-fluid layer subject to a uniform magnetic field. A few recent studies have shown that a non-uniform MR-fluid treatment could be beneficial in limiting the deflection response for a transverse excitation [Lara-Prieto et al., 2010; Haiqing et al., 1993; Haiqing and King, 1997; Rajamohan et al., 2010b]. The above studies, however, employed distinctly different approaches. Prieto et al. (2010) experimentally investigated the dynamic responses of a MR-sandwich cantilever beam subject to a uniform and non-uniform magnetic field. The non-uniform magnetic field was realized by distributing five sets of permanent magnets over the entire surface of the beam, while the field intensity of each magnet was identical. It was concluded that the natural frequency of the beam decreases as the permanent magnets are moved away from the fixed support. The study also investigated the dynamic responses of sandwich beams with two different face materials, Aluminum and polyethylene terephthalate (PET) and concluded that PET plates could provide relatively higher changes in natural frequencies with applied magnetic field. Alternatively, Haiqing et al. $(1993,1997)$ and Rajamohan et al. (2010b) proposed partial ER/MR-fluids treatments, respectively, by introducing a number of local fluid segments over the span of the beam. 
Haiqing et al. (1993) experimentally analyzed the vibration characteristics of a cantilever beam with ER fluid applied only at the mid-section of the beam, which was coupled to the ground. The experimental study showed that the locally applied ER fluid could serve as a complex spring that would alter the damping and stiffness properties of the structures significantly under an electric field. The vibration response of a clamped-clamped beam with three ER fluid segments separated by an air cavity over the beam length was also investigated experimentally by Haiqing and King (1997). The results showed that the length of the ER fluid segments strongly influence the resonant frequencies and the loss factors. Rajamohan et al. (2010b) presented finite element formulations for a partially-treated MR fluid sandwich beam comprising various MR-fluid segments for different boundary conditions. The free and forced vibration responses of different configurations of partially-treated MR-fluid beams were derived for various lengths and number of fluid segments. The study also performed laboratory experiments to demonstrate validity of the analytical formulations and concluded that the location and length of the MR fluid segments have significant effect on the natural frequencies and the loss factors, in addition to the intensity of the magnetic field and the boundary conditions. The influence of locations of the MR fluid segments on the modal damping factor was further investigated under different end conditions using modal strain energy approach and finite element method by Rajamohan et al. (2010c). Optimal configurations of a partially treated MR sandwich beam were subsequently identified to achieve maximum modal damping factor corresponding to the first five flexural modes, considered either individually or simultaneously.

The vibration properties of MR/ER-fluid treated beams have been mostly investigated under various fixed intensities of the applied field in an open-loop manner. The efforts in deriving semi-active and active control synthesis have been mostly limited to simple single- or two-degree of freedom lumped parameters models, where the stiffness and/or damping properties are described as a function of the applied field [Leitmann and Reithmeier, 1993; Leitmann, 1994]. Only limited efforts have been made towards synthesis of semi-active and active controllers for the ER/MR-fluid treated sandwich beams, although the controller design for structures employing piezoelectric actuators have been widely reported (Sadri et al., 1999; Hu and Ma, 2005; Baillargeon and Vel, 2005). Shaw (2000) proposed a two-stage controller to reduce the vibration of a ER-fluid beam subjected to harmonic excitations and investigated the performance characteristics through laboratory experiments. The study employed two independent controllers: a fuzzy logic-based semi-active controller to tune the resonance frequencies; and an active force control for cancelling the external disturbance. Representation of the finite element modeling of the MR sandwich beam into state space form as a function of magnetic field and development of a closed loop semi-active control synthesis to control the dynamic characteristics of the beam, however, have not yet been explored.

In the present study, a semi active control synthesis is derived to control the dynamic characteristics of the fully and partially treated MR sandwich beams. The governing equations of motion of the three layer MR sandwich beam formulated in the finite element form are expressed in the state variable form, and an observer-based linear quadratic regulator (LQR) optimal control strategy is developed. A reduced-state controller synthesis is further derived on the basis of the estimated flexural mode shapes (FMS) of the beam. The effectiveness of the reduced-state control is demonstrated by comparing the vibration responses with those of the beam with the full-state LQR control. Simulations are performed by using both observer and FMS based LQR control strategies to investigate the tip displacement response of the fully and partially treated MR sandwich beams under impulse and white noise disturbances.

\section{Finite element formulation of MR sandwich beam}

The finite element formulations for the fully and a partially treated three layer beam structures containing MR fluid as the core in between the two elastic layers, as shown in Figure 1 have been reported in [Rajamohan et al., 2010a] and [Rajamohan et al., 2010b], respectively. These formulations are considered in this study for synthesis of a semi-active vibration control of the multilayer beam. 


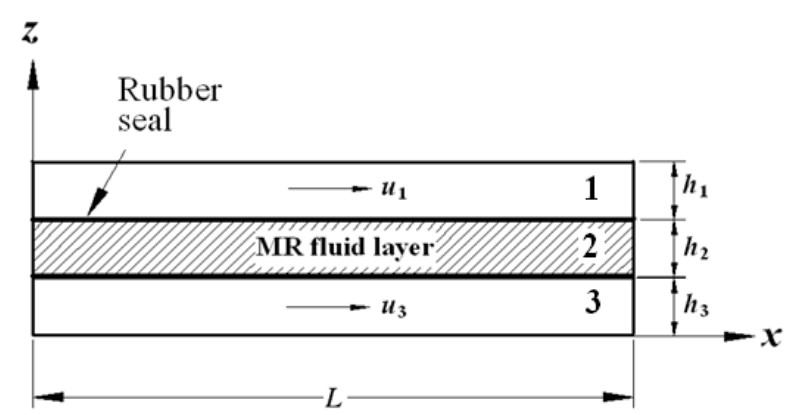

(a)

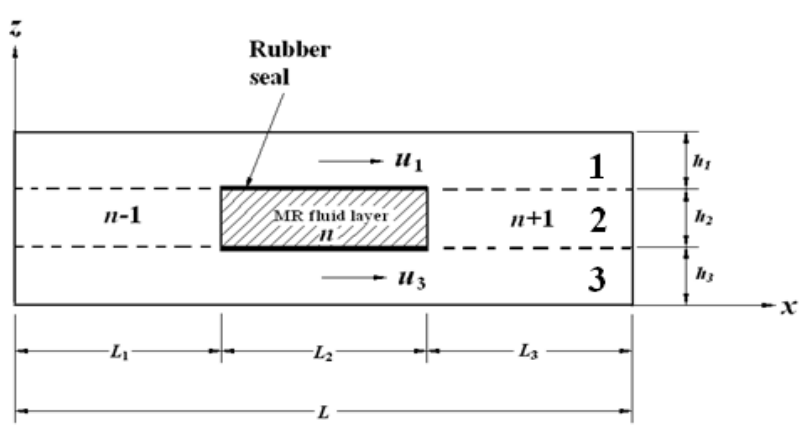

(b)

Figure 1. (a) Fully treated MR sandwich beam. (b) Partially treated MR sandwich beam

Energy expressions for a fully and a partially treated MR sandwich beam have been first derived. The governing equations of motion in the finite element form are then derived using Lagrange's energy approach upon by considering a two-node beam element with three degrees of freedom (transverse $w$, axial $u$ and rotational $\theta$ displacements of the beam) at each node and are represented as follows:

$$
\left[m^{e}\right]\{\ddot{d}\}+\left[k^{e}\right]\{d\}=\left\{f^{e}\right\}
$$

where $\left[m^{e}\right]$ and $\left[k^{e}\right]$ are the element mass and stiffness matrices, respectively, and $\left\{f^{e}\right\}$ is the element force vector and $\{d\}$ is the displacement vector. The detailed formulation of $\left[k^{e}\right]$ and $\left[m^{e}\right]$ can be found in [Rajamohan et al., 2010a, 2010b]. Assembling the mass and stiffness matrices and the force vector for all the elements yields the system governing equations of motion of the MR sandwich beam in the following form:

$$
[M]\{\ddot{d}\}+[K]\{d\}=\{F\}
$$

where $[M],[K]$ and $\{F\}$ are the system mass and stiffness matrices, and the force vector, respectively. The matrix $[K]$ is a complex stiffness matrix which includes both stiffness of the structure and damping attributed to the complex shear modulus of the MR fluid layer.

\section{Design of an optimal controller}

Control of vibration in a structure is generally concerned with specific modes of flexural vibration. It may thus be appropriate to express (2) in the modal form using the modal coordinates, which would yield uncoupled governing equations of motion for the MR sandwich beam. Assuming proportional damping, (2) can be written in the following form:

$$
\left\{\ddot{\eta}_{i}\right\}+\left[2 \xi_{i} \omega_{i}\right]\left\{\dot{\eta}_{i}\right\}+\left[\omega_{i}^{2}\right]\left\{\eta_{i}\right\}=\left\{f_{i}\right\}, i=1,2, \ldots, n
$$

where $\{\eta\}$ is the modal coordinate vector, which is related to the modal matrix $[q]$ such that $\{d\}=[q]\{\eta\}$, $\xi_{i}$ is the modal damping ratio for the $i^{\text {th }}$ normal mode, $\omega_{i}$ is the corresponding natural frequency of the system without considering the structural damping, and $f_{i}=[q]^{T}\{F\}$. Both the damping factor and natural frequencies of the beam would vary with the applied magnetic field; (3) may thus be expressed as a function of the controlled magnetic field $u_{i}$ :

$$
\left\{\ddot{\eta}_{i}\right\}+C^{\prime}\left(u_{i}\right)\left\{\dot{\eta}_{i}\right\}+K^{\prime}\left(u_{i}\right)\left\{\eta_{i}\right\}=\left\{f_{i}\right\} ; i=1,2, \ldots, n
$$

where $K^{\prime}\left(u_{i}\right)=\left[\omega_{i}^{2}\right]$ and $C^{\prime}\left(u_{i}\right)=\left[2 \xi_{i} \omega_{i}\right]$. 

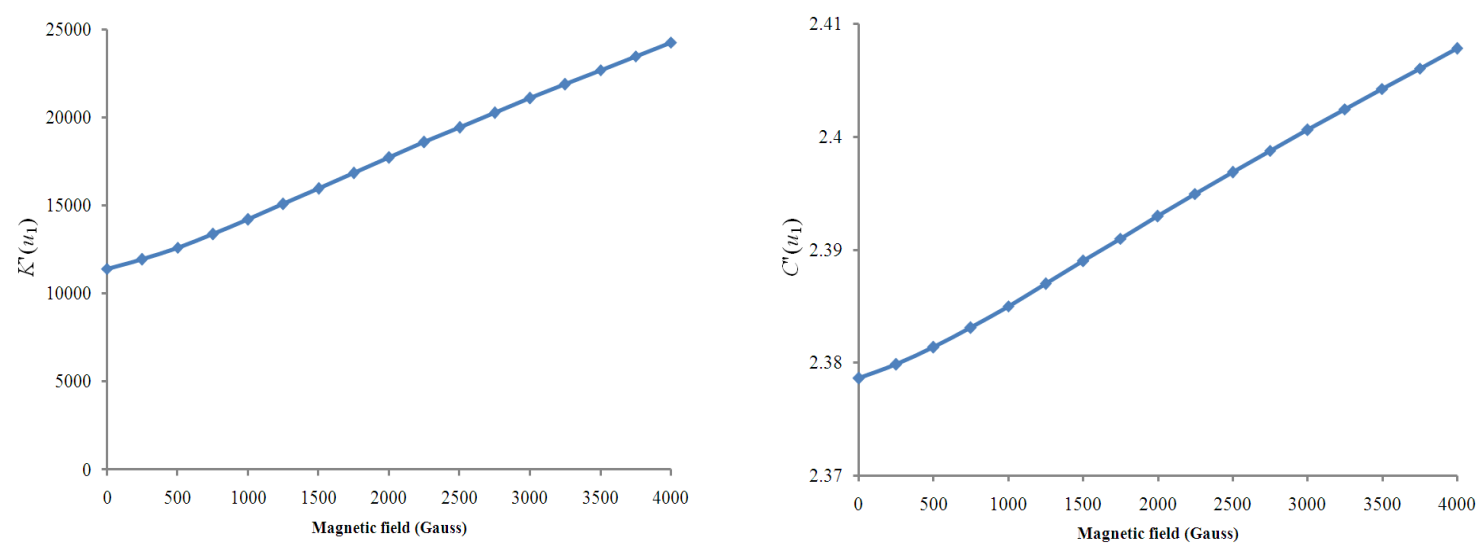

Figure 2. Variation of $K^{\prime}\left(u_{i}\right)$ and $C^{\prime}\left(u_{i}\right)$ with magnetic field at Mode 1

It has been shown that the natural frequencies of the sandwich beam vary linearly with the applied magnetic field (Rajamohan et al., 2010a). The variation of the factors, $K^{\prime}\left(u_{i}\right)$ and $C^{\prime}\left(u_{i}\right)$, with the applied magnetic field in range of 0 - 4000 Gauss is investigated for various modes of vibration and the results showed that both factors vary approximately linearly with the magnetic field. For instance for mode 1, the variation of $K^{\prime}\left(u_{i}\right)$ and $C^{\prime}\left(u_{i}\right)$ with respect to the applied magnetic field is shown in Figure 2, which clearly demonstrates that these factors nearly vary linearly with respect to the applied magnetic field. Hence for the sack of simplicity $K^{\prime}\left(u_{i}\right)=\left[\omega_{i}^{2}\right]$ and $C^{\prime}\left(u_{i}\right)=\left[2 \xi_{i} \omega_{i}\right]$ are assumed to be linear functions of the applied magnetic field $u_{i}$ such that:

$$
K^{\prime}\left(u_{i}\right)=\alpha_{k i}+\beta_{k i} u_{i}, 0 \leq u_{i} \leq u_{\max } ; C^{\prime}\left(u_{i}\right)=\alpha_{c i}+\beta_{c i} u_{i}, 0 \leq u_{i} \leq u_{\max }
$$

where $u_{\max }$ refers to the maximum magnetic field that could be applied, which may be limited due to the practical limitations of the electromagnet, power requirement and/or the saturation limit of the MR fluid used. The coefficients $\alpha_{k i}, \beta_{k i}$ and $\alpha_{c i}, \beta_{c i}$ are functions of the applied magnetic field corresponding to each mode, $u_{i}$. In this study, a linear quadratic regulator (LQR) based controller synthesis is realized using the full state feedback. The finite element models of the fully- and partially- treated beams are thus expressed in the state-space form, as:

$$
\left\{\dot{x}_{S}\right\}=[A]\left\{x_{S}\right\}+[B] \gamma+\{f\} \text { and }\left\{y_{S}\right\}=[C]\left\{x_{S}\right\}
$$

where $[A]=\left[\begin{array}{cc}{[0]} & {[I]} \\ {\left[-\alpha_{k i}\right]} & {\left[-\alpha_{c i}\right]}\end{array}\right] ;[B]=\left[\begin{array}{cc}{[0]} & {[0]} \\ {\left[-\beta_{k i}\right]} & {\left[-\beta_{c i}\right]}\end{array}\right] ;[C]=\left[\begin{array}{cc}I & 0 \\ 0 & 0\end{array}\right] ;\{\gamma\}=[U]\left\{x_{S}\right\}[U]=\left[\begin{array}{cc}{\left[u_{i}\right]} & {[0]} \\ {[0]} & {\left[u_{i}\right]}\end{array}\right]$

is the control input matrix; $\{f\}=\left[\begin{array}{c}{[0]} \\ {\left[f_{i}\right]}\end{array}\right]$; and $\left\{x_{s}\right\}=\{\{\eta\},\{\dot{\eta}\}\}^{T}$ is the state vector; and $\left\{y_{s}\right\}$ is the

response vector.

In (6), the generalized excitation force vector $\{f\}$ and the matrix $[U]$ define the inputs, while $\left\{y_{S}\right\}$ is the output vector. In the closed-loop configuration, the control input vector $\{\gamma\}$ in (6) is related to the state feedback vector, as:

$$
\{\gamma\}=-[U]\left\{x_{S}\right\}
$$

where the magnetic field matrix $[U]$ serves as the control gain matrix, which is evaluated according to the desired control law, while negative sign indicates externally applied control magnetic field. Upon substituting for $\{\gamma\}$ in (6), the closed loop system can be expressed in the state space form, as:

$$
\left\{\dot{x}_{S}\right\}=([A]-[B][U])\left\{x_{S}\right\}+\{f\}
$$

From (5), it can be stated that the control gain matrix $[U]$ directly relates to the system damping, $C^{\prime}\left(u_{i}\right)$, and stiffness, $K^{\prime}\left(u_{i}\right)$, properties. An optimal control is synthesized through minimization of a cost function 
that is proportional to a measure of the system's response and the desired control inputs using the linear quadratic regulator (LQR) approach [Sethi and Song, 2005; Burl, 1999], such that:

$$
J=\frac{1}{2} \int_{0}^{\infty}\left(\left\{x_{S}\right\}^{T}[Q]\left\{x_{S}\right\}+\{\gamma\}^{T}[R]\{\gamma\}\right) d t
$$

where $[Q]$ and $[R]$ are the symmetric semi-definite and positive-definite weighting matrices, respectively. The relative magnitudes of $[Q]$ and $[R]$ are selected so as to achieve an optimal trade off between the vibration response and the intensity of the control magnetic field. While $[Q]$ defines the relative weight of each state variable, $[R]$ defines the relative weight of control magnetic field [Liao et al, 2003 and Varadharajan et al, 2000].

Minimization of (9) yields a linear full-state feedback control law, $\{\gamma\}=-[U]\left\{x_{S}\right\}$, where the control gain matrix, $[U]=[R]^{-1}[B]^{T}[P]$ is evaluated by solving for $[P]$ from the following algebraic Ricatti equation [Mutambara, 1999]:

$$
[A]^{T}[P]+[P][A]+[Q]-[P][B][R]^{-1}[B]^{T}[P]=0
$$

\subsection{Observer based optimal controller}

The LQR controller based on the full state dynamic observer is formulated to derive the control gain matrix [Burl, 1999; Mutambara, 1999]. The dynamic state observer can be given by:

$$
\left\{\hat{\hat{x}}_{S}\right\}=[A]\left\{\hat{x}_{S}\right\}+[B]\{\gamma\}+[L]\left(\left\{y_{S}\right\}-\left\{\hat{y}_{S}\right\}\right)+\{f\} ; \text { and } \quad\left\{\hat{y}_{S}\right\}=[C]\left\{\hat{x}_{S}\right\}
$$

where $\left\{\hat{x}_{S}\right\}$ is the estimated state vector and $[L]$ is the observer gain evaluated based on LQR control law and determines the convergence of $\left\{x_{S}\right\} \rightarrow\left\{\hat{x}_{S}\right\}$, and $\left\{\hat{y}_{S}\right\}$ is the output vector evaluated from $\left\{\hat{x}_{S}\right\}$.

The control input vector can also be expressed as a function of $\left\{\hat{x}_{S}\right\}$ such that: $\{\gamma\}=-[U]\left\{\hat{x}_{S}\right\}$.

Eqs. (6) and (11) may be written in terms of the estimated state, as:

$$
\begin{aligned}
& \left\{\dot{x}_{S}\right\}=[A]\left\{x_{S}\right\}-[B][U]\left\{\hat{x}_{S}\right\}+\{f\} \\
& \left\{\dot{x}_{S}\right\}=([A]-[B][U]-[L]\{C])\left\{\hat{x}_{S}\right\}+[L]\left\{y_{S}\right\}+\{f\}
\end{aligned}
$$

Upon substituting for $\left\{y_{S}\right\}=[C]\left\{x_{S}\right\}$ in (13) and rearranging, the closed loop system with observer yields:

$$
\left\{\begin{array}{c}
\dot{x}_{S} \\
\dot{\hat{x}}_{S}
\end{array}\right\}=\left[\begin{array}{cc}
{[A]} & -[B][U] \\
{[L][C]} & {[A]-[B][U]-[L][C]}
\end{array}\right]\left\{\begin{array}{l}
x_{S} \\
\hat{x}_{S}
\end{array}\right\}+\left\{\begin{array}{l}
f \\
f
\end{array}\right\}
$$

Equation (12) can also be rewritten in terms of the observer error $\mathcal{E}$ between the measured and estimated state vector, as:

$$
\left\{\dot{x}_{S}\right\}=([A]-[B][U])\left\{x_{S}\right\}+[B][U] \varepsilon+\{f\}
$$

where $\varepsilon=\left\{x_{S}\right\}-\left\{\hat{x}_{S}\right\}$, which can be derived from Eqs.(13) and (8), as:

$$
\dot{\varepsilon}=([A]-[B][U]-[L][C]) \mathcal{E}+\{f\}
$$

The closed loop system with the observed state vector feedback control system can be expressed using the above two equations, as:

$$
\left\{\begin{array}{c}
\dot{x}_{S} \\
\dot{\varepsilon}
\end{array}\right\}=\left[\begin{array}{cc}
{[A]-[B][U]} & {[B][U]} \\
0 & {[A]-[B][U]-[L][C]}
\end{array}\right]\left\{\begin{array}{c}
x_{S} \\
\varepsilon
\end{array}\right\}+\left\{\begin{array}{l}
f \\
f
\end{array}\right\}
$$

The above closed-loop system with observer based LQR controller is known to be inherently stable, while the observability and controllability of the system have also been evaluated through solution of the Lyapunov equations for controllability gramian $\left[W_{C}\right]$ and observability gramian $\left[W_{O}\right]$, given by [Ogata, 2008]:

$$
[A]\left[W_{C}\right]+\left[W_{C}\right]\left[A^{T}\right]+[B]\left[B^{T}\right]=0 ; \text { and }\left[A^{T}\right]\left[W_{O}\right]+\left[W_{O}\right][A]+\left[C^{T}\right][C]=0
$$

The system is said to be controllable and observable if $\left[W_{C}\right]$ and $\left[W_{O}\right]$ are of full rank. 


\subsection{Flexural mode shape (FMS) based optimal controller}

Unlike the full-state LQR control, a limited state control synthesis could be realized using the mode shapes of the flexible beam. This approach could considerably reduce the number of feedback variables and facilitate hardware implementation by limiting the number of sensors. The known flexural mode shapes of the beam could be applied to obtain estimates of the state vector on the basis of measurements of only a few state variables. The flexural mode shape (FMS) based controller design can be further simplified, when the vibration control is sought for only a few selected modes. Using the mode summation method, the displacement response vector of the beam can be estimated from the flexural mode shapes, as:

$$
\left\{d_{e}(x, t)\right\}=\sum_{i=1}^{n}\left\{\phi_{i}(x)\right\} q_{i}(t)
$$

where $\left\{d_{e}(x, t)\right\}$ is the estimated displacement vector of the beam, $\left\{\phi_{i}(x)\right\}$ is the mode shape vector corresponding to the normal mode $i$ and $q_{i}$ is the generalized $i^{\text {th }}$ coordinate. Considering that the vibration response of a beam is generally dominated by a few lower modes, the limited state controller synthesis is formulated by considering only the first two modes. The estimation of the generalized coordinate vectors corresponding to the first two modes, $\left\{q_{1}(t)\right\}$ and $\left\{q_{2}(t)\right\}$, would require measurements of the state at a minimum of two locations. Initially the mode shape vector corresponds to the two modes, $\phi_{1}$ and $\phi_{2}$, could be obtained for the open-loop structure in the absence of a magnetic field. Considering the measurements of deflections, $d_{m}(l / 2)$ and $d_{m}(l)$ at $x=l / 2$ and $x=l$, respectively, the generalized coordinates may be estimated from:

$$
\left\{\begin{array}{c}
q_{1}(t) \\
q_{2}(t)
\end{array}\right\}=\left[\begin{array}{cc}
\phi_{1}(l) & \phi_{2}(l) \\
\phi_{1}(l / 2) & \phi_{2}(l / 2)
\end{array}\right]^{-1}\left\{\begin{array}{c}
d_{m}(l, t) \\
d_{m}(l / 2, t)
\end{array}\right\}
$$

Equation (19) yields the estimated displacement vector $\left\{d_{e}(x, t)\right\}$ on the basis of two measured deflections, which is subsequently used to evaluate the control vector, $\{\gamma\}=-[U]\left\{x_{e}(x, t)\right\}$, where the estimated state vector $\left\{x_{e}(x, t)\right\}$ consists of $\left\{d_{e}(x, t)\right\}$ and their derivatives. The equation for the closed loop system based on FMS optimal controller can thus be written as:

$$
\left\{\dot{x}_{S}\right\}=([A]-[B][U])\left\{x_{e}(x, t)\right\}+\{f\}
$$

\section{RESULTS AND DISCUSSION}

The fundamental characteristics of the fully-and partially-treated MR sandwich beam were investigated theoretically and experimentally and reported in Rajamohan et al. (2010a) and Rajamohan et al. (2010b), respectively. The permanent magnets were used in experimental set-up to generate the desired magnetic field over the surface of the MR sandwich beam by varying the gap between the magnets and the structure. The realization of a controllable MR sandwich structure, however, would require designs of compact electro-magnets in order to apply the desired field over the surface of the beam. The designs of compact electro-magnets have been realized for MR fluid dampers employed in vehicle suspensions, where the electro-magnet is integrated within the damper piston [Dyke et al., 1998]. The design of electromagnets for MR sandwich structures, however, involves additional challenges considering the larger size of the structure compared to the damper piston. Therefore, further efforts are needed to design compact yet high intensity electromagnets for practical implementation of the controller. This study, however, is limited to simulations to evaluate the effectiveness of the developed full-and limited-state optimal LQR controllers in suppressing the vibration of the fully- and partially-treated MR sandwich beam with clamped-free boundary conditions under a unit impulse load and a white noise force disturbance applied at the tip of the beam. A white noise force signal was synthesized to yield nearly constant auto spectral density up to $500 \mathrm{~Hz}$. The simulation results were obtained by considering identical baseline thickness $(1 \mathrm{~mm})$ of each of the elastic and fluid layers, while the length and width of the fully- 
and partially-treated beams were taken as $300 \mathrm{~mm}$ and $30 \mathrm{~mm}$, respectively. The material properties were considered as: $\rho_{1}=\rho_{3}=2700 \mathrm{~kg} / \mathrm{m}^{3} ; E_{1}=E_{3}=68 \mathrm{GPa} ; \rho_{r}=1233 \mathrm{~kg} / \mathrm{m}^{3}$ and $\rho_{2}=3500 \mathrm{~kg} / \mathrm{m}^{3}$, where $\rho_{1}$ and $\rho_{3}$ are the mass densities of the elastic layers, and $\rho_{2}$ and $\rho_{r}$ are the mass densities of the fluid and the sealant rubber material, respectively. $E_{1}$ and $E_{3}$ are Young's moduli of the elastic layers 1 and 3, respectively. Two different partially-treated MR sandwich beams were configured with four localized MR fluid segments, each having $20 \mathrm{~mm}$ length. The locations of the fluid segments were chosen on the basis of an earlier study [Rajamohan et al., 2010c], and denoted as configurations A and configuration B, as shown in Figures 3(a) and 3(b), respectively. The configurations A and B were shown to yield maximum damping factors corresponding to the first five modes, when subjected to a constant magnetic field [Rajamohan et al., 2010c]. Furthermore, the complex shear modulus of the MR fluid was considered to be a function of the storage and loss moduli, as [Rajamohan et al., 2010a]:

$$
G^{*}(B)=G^{\prime}(B)+i G^{\prime \prime}(B)
$$

where $G^{\prime}$ and $G^{\prime \prime}$ are storage and loss moduli, respectively, expressed as the nonlinear functions of the magnetic field intensity $B$.

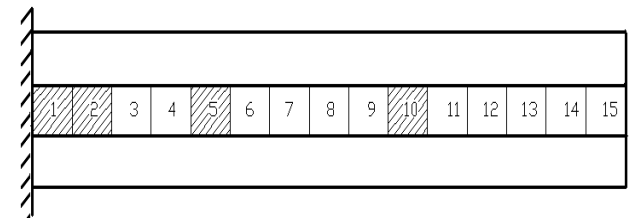

(a)

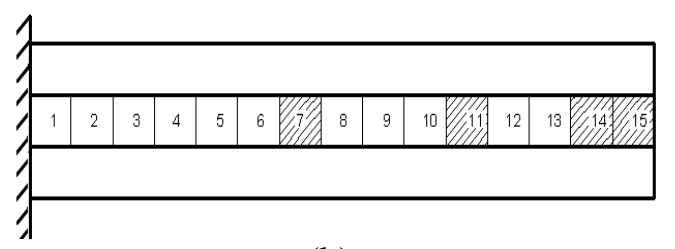

(b)

Figure 3. Selected configurations of partially treated MR sandwich beams with maximum modal damping factors corresponding to first five modes under a constant magnetic field:

(a) configuration $\mathrm{A}$; and (b) configuration $\mathrm{B}$.

\subsection{Full-state observer based LQR control}

\subsubsection{Response to impulse disturbance}

The free vibration response of the closed-loop fully-and partially- treated beams were evaluated under an impulse force applied at the free end of the clamped-free beam. The results are presented to illustrate the effectiveness of the LQR controller design described in (17). The weighting matrices $[Q]$ and $[R]$ of the controller were identified through minimization of a composite performance function of the peak displacement along the $z$-axis and the settling time, such that

$$
\text { Minimize } f(x)=\alpha_{1} t_{s}+\alpha_{2} d_{z}(l)
$$

where $t_{s}$ is the settling time, $d_{z}(l)$ is the deflection at the tip along the $z$-axis (transverse deflection), and $\alpha_{1}$ and $\alpha_{2}$ are the constant weighting factors. The settling time was identified as the displacement responses of the beam reach the order of $10^{-6} \mathrm{~m}$. The above minimization problem was solved using the sequential quadratic programming (SQP) method available in optimization toolbox [Matlab ${ }^{\circledR}$ ], where the maximum field intensity was limited, $B \leq 4000$ Gauss. Limit constraints were also imposed on $[R]$ and $[Q]$, as

$$
\left[R_{l} \leq[R] \leq\left[R_{u}\right] ; \text { and }\left[Q_{l}\right] \leq[\mathrm{Q}] \leq\left[\mathrm{Q}_{u}\right]\right.
$$

The limiting values, $\left[R_{l}\right],\left[R_{u}\right],\left[Q_{l}\right]$ and $\left[\mathrm{Q}_{u}\right]$, were identified through a parametric study involving the effects of variations in $[R]$ and $[Q]$ on $t_{s}$ and $d_{z}(l)$ together with the required field intensity. The lower and upper bounds were identified as the values when the magnetic field, $B$, exceeded 4000 Gauss, which are summarized in Table 1 together with the optimal values $\left[R^{*}\right]$ and $\left[Q^{*}\right]$ for the fully-and partially-treated beams obtained from the solutions of the minimization problem in (24). It should be noted that the minimization problem was solved assuming different weighting factors ranging from 0 to $1\left(\alpha_{1}+\alpha_{2}=1\right)$ and different values of the starting vectors. All of the solutions converged to very similar values of $[R]$ and $[Q]$. 
Table 1. The lower and upper bounds of $[\mathrm{R}]$ and $[\mathrm{Q}]$ and the optimal values, $\left[\mathrm{R}^{*}\right]$ and $\left[\mathrm{Q}^{*}\right]$

\begin{tabular}{ccccccc}
\hline Beam & {$\left[R_{l}\right]$} & {$\left[R_{\mathrm{u}}\right]$} & {$\left[Q_{l}\right]$} & {$\left[Q_{\mathrm{u}}\right]$} & {$\left[Q^{*}\right]$} & {$\left[R^{*}\right]$} \\
\hline Fully treated MR sandwich beam & 10 & 100 & 100 & 2500 & 1390 & 39.45 \\
Configuration A & 10 & 60 & 100 & 3000 & 2690 & 50.45 \\
Configuration B & 10 & 175 & 100 & 2500 & 2300 & 151.5 \\
\hline
\end{tabular}

The time-history and the frequency spectra of the tip deflection responses of the controlled fullytreated MR sandwich beam to an impulse excitation are compared with those of the passive beam $(B=0)$ in Figures 4(a)-4(c). The time-history of the required control magnetic field is also shown in Figure 4(d). From the results, it is evident that the LQR control algorithm can significantly attenuate the tip displacement with the permissible intensity of the control magnetic field. The results show that the settling time of the controlled beam is in the order of $0.59 \mathrm{~s}$, which is significantly lower than $4.325 \mathrm{~s}$ of the passive beam. The amplitude spectra of the tip displacement response, illustrated in Figure 4(c), also shows substantially lower deflections of the controlled beam corresponding to all of the modes observed upto $250 \mathrm{~Hz}$.

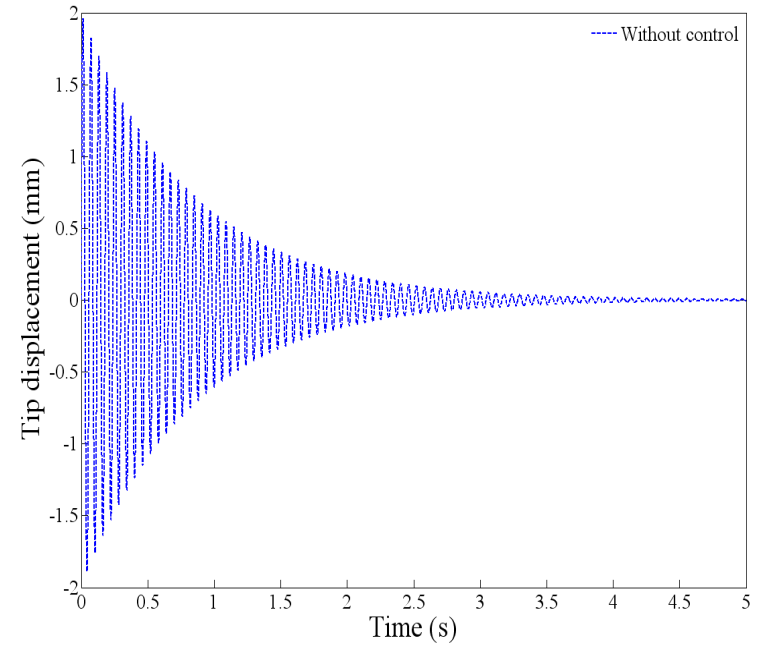

(a)

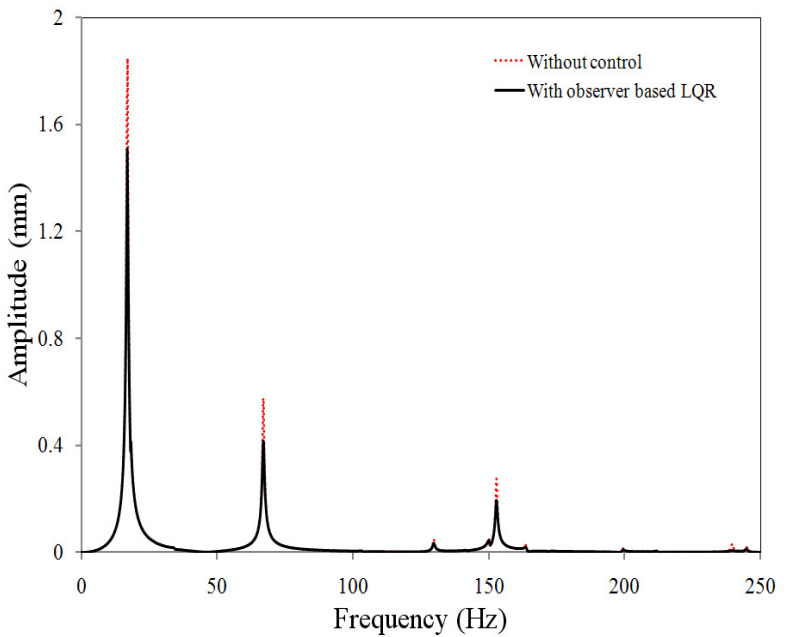

(c)

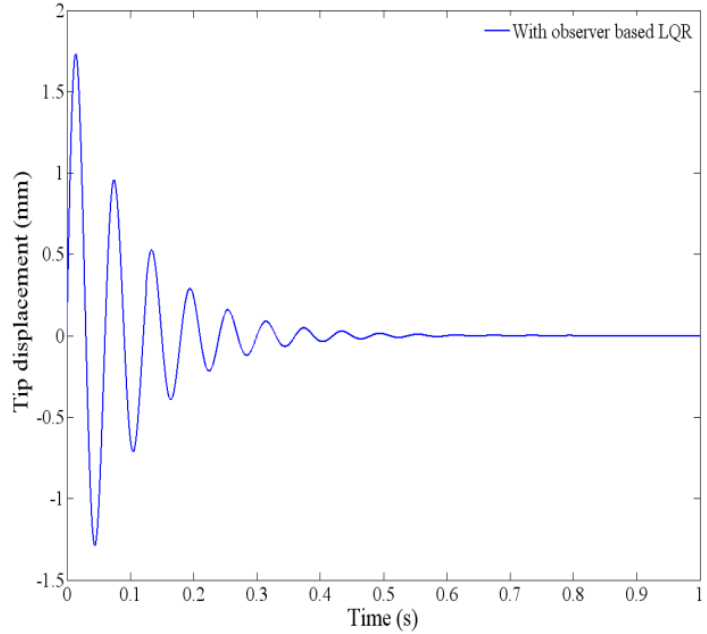

(b)

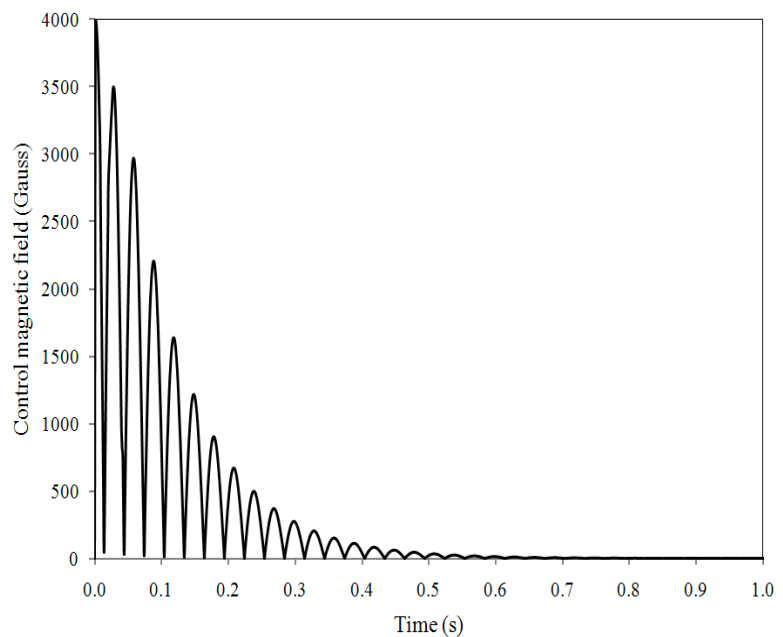

(d)

Figure 4. The tip deflection responses of the fully-treated MR sandwich beam with and without the LQR control and subject to a unit load impulse: (a) time-history: without control; (b) time-history: with observer based control; (c) amplitude spectrum; (d) time-history of control magnetic field. 
The results show that the amplitudes corresponding to the first three natural frequencies of the controlled fully-treated beam are 18\%, 28\% and 32\%, respectively, lower than those of the uncontrolled beam. The natural frequencies of the controlled beam, however, are quite close to those of the passive beam. Although it has been shown that the application of a constant magnetic field significantly alters the stiffness property of the MR beam [Rajamohan et al., 2010a], the results in Figure 4 do not show a notable change in the natural frequencies corresponding to the first three modes. This is mostly attributed to the rapid settling time of the controlled beam, where the control magnetic field vanishes at $t>0.5 \mathrm{~s}$, as seen in Figure 4(d).

Figures 5 (a) and 5 (b) show the time-histories and amplitude spectra, respectively, of free vibration responses of the partially treated sandwich beams (configuration A) with and without the observer-based controller, evaluated at the tip. The figures compare the responses of the controlled and passive $(B=0)$ partially-treated beams subject to an impulse unit load. The results show substantially lower settling time of the partially treated beam employing the LQR control algorithm compared to that of the passive beam. The settling time of the controlled partially treated beam is $0.53 \mathrm{~s}$, which is slightly lower than that of the fully treated beam $(0.59 \mathrm{~s})$. The deflection amplitudes of the partially treated beam corresponding to the first three modes, however, are significantly greater than those of the fully treated beam, but lower than those of the respective passive beam. The deflection amplitudes of the controlled partially-treated beam corresponding to the first three modes are $14 \%, 15 \%$ and $9 \%$ lower than those of the uncontrolled beam. A further comparison of Figures 4(c) and 5(b) suggests considerable difference in the natural frequencies of the fully-and partially-treated beams. These are attributable to the differences in their structures, particularly the density of the MR fluid and the elastic material $\left(\rho_{2}>\rho_{1}\right)$. The total mass of the fully treated beam is thus relatively higher. The observed frequencies of both the fully-and partiallytreated beams were identical to those reported in [Rajamohan et al., 2010a, 2010b].

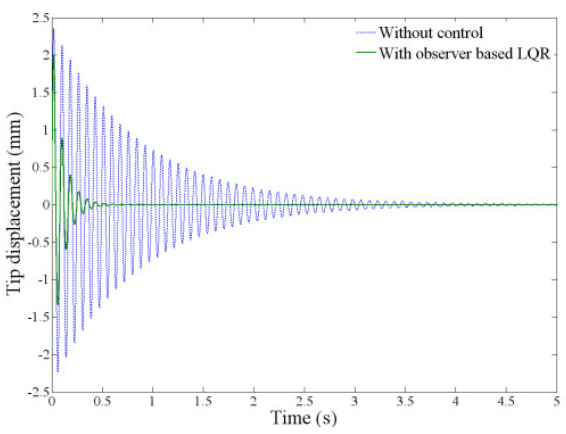

(a)

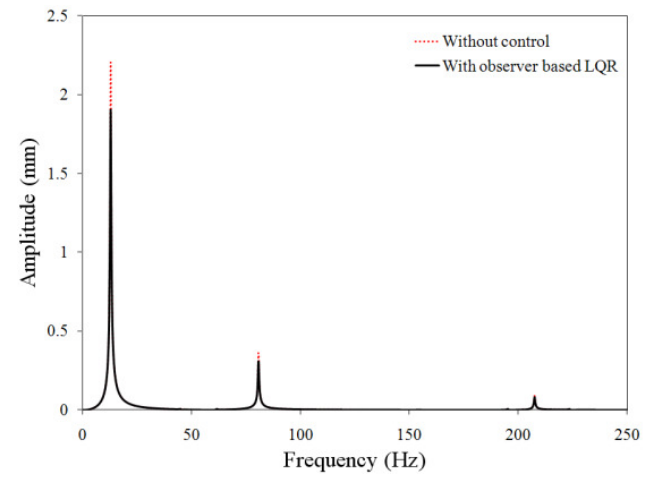

(b)

Figure 5. The tip deflection responses of the partially-treated MR sandwich beam (configuration A) with and without the observer based LQR control and subject to a unit load impulse: (a) time-history; (b) amplitude spectrum.

The time-histories and amplitude spectra of tip displacement responses of configuration B of the controlled as well as passive partially-treated sandwich beams are illustrated in Figures 6(a) and 6(b), respectively. The greater treatment near the free end yields larger reduction in the tip displacement, while the settling time tends to be slightly higher for both the controlled as well as passive beams compared to the fully-treated and configuration A of the partially-treated beams. The implementation of the LQR control algorithm yields peak tip displacement of $1.45 \mathrm{~mm}$ and settling time of $0.62 \mathrm{~s}$. The corresponding values for the passive beam are $1.77 \mathrm{~mm}$ and $4.4 \mathrm{~s}$, respectively. The amplitudes corresponding to the first three natural frequencies of the controlled structure are $15 \%, 41 \%$ and $56 \%$, respectively, lower than those of the passive beam. The deflection amplitudes of the controlled configuration B are substantially lower than those of the controlled configuration A. This is particularly attributable to relative higher modal damping factors of configuration B [Rajamohan et al., 2010c]. Furthermore, the peak displacement 
response at the tip of the passive partially-treated beam (configuration B) is almost comparable to that of the fully treated passive beam, as observed from the time-history of the responses. Similar trend has also been reported in [Rajamohan et al., 2010a, 2010b] in the transverse response of the fully and various configurations of the partially treated beams. The results also show that the implementation of the LQR control to configuration $\mathrm{B}$ yields significantly higher reductions in deflection amplitudes corresponding to the first three modes compared to those of observed for the fully treated and configuration $\mathrm{A}$ of the partially treated beam.

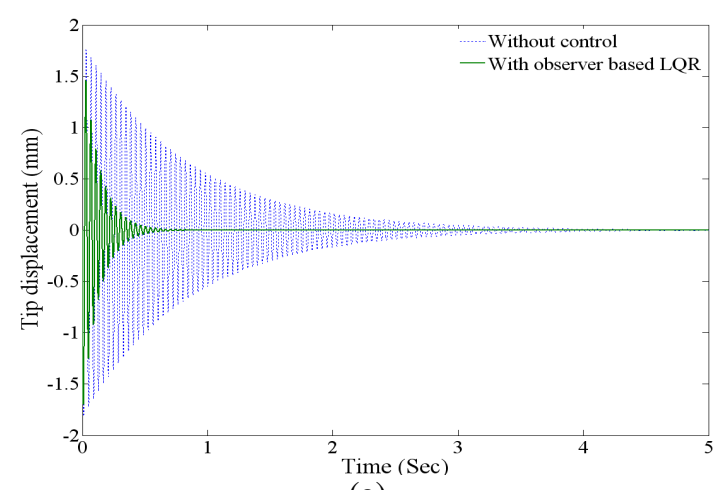

(a)

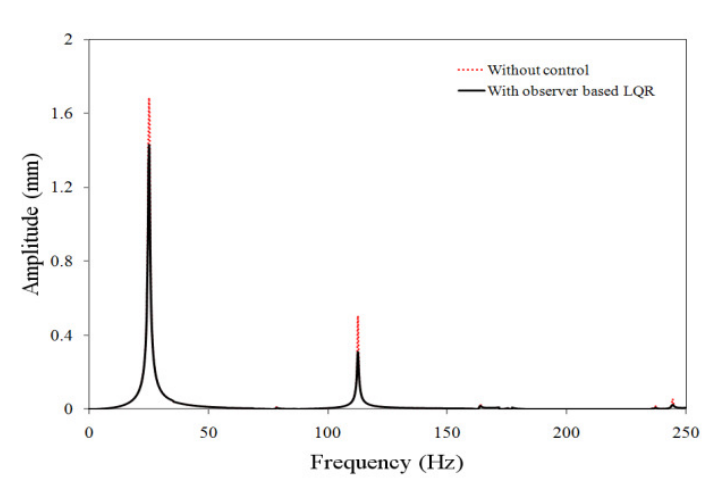

(b)

Figure 6. The tip deflection responses of the partially-treated MR sandwich beam (configuration B) with and without the observer based LQR control and subject to a unit load impulse: (a) time-history; (b) amplitude spectrum.

\subsubsection{Response to a white noise disturbance}

The effectiveness of the LQR control in suppressing the steady-state vibration is further evaluated under a Gaussian white noise force disturbance applied at the tip along $z$-axis (variance $=3.15 \mathrm{~N}^{2}$; mean $\approx 0$ ). The optimal state weighing matrices, $\left[R^{*}\right]$ and $\left[Q^{*}\right]$, were subsequently evaluated in a similar manner through solution of the following minimization problem:

$$
\text { Minimize, } f(x)=\sum_{i=1}^{n} d_{z p_{i}}(l)
$$

The above minimization problem considers the peak transverse deflection, $d_{z p_{i}}(l)$, at the tip corresponding to the first five modes $(n=5)$. The peak deflections corresponding to different modes were extracted from the amplitude spectrum of the tip deflection response by defining five distinct frequency ranges around the known natural frequencies of the passive beam $(\mathrm{B}=0)$. The lower and upper bounds of $[R]$ and $[Q]$ were obtained from a parametric study as in the case of impulse excitation, and are summarized in Table 2 together with the optimal values, $\left[R^{*}\right]$ and $\left[Q^{*}\right]$ for the fully- and partially-treated beams.

Table 2. The lower and upper bounds of $[\mathrm{R}]$ and $[\mathrm{Q}]$ and the optimal values, $\left[\mathrm{R}^{*}\right]$ and $\left[\mathrm{Q}^{*}\right]$

\begin{tabular}{ccccccc}
\hline Beam & {$\left[R_{l}\right]$} & {$\left[R_{u}\right]$} & {$\left[Q_{l}\right]$} & {$\left[Q_{\mathrm{u}}\right]$} & {$\left[Q^{*}\right]$} & {$\left[R^{*}\right]$} \\
\hline Fully treated MR sandwich beam & 43 & 45 & 4000 & 4300 & 4125 & 43.25 \\
Configuration A & 25 & 65 & 1750 & 3500 & 2800 & 51.5 \\
Configuration B & 25 & 65 & 1750 & 3500 & 2800 & 51.5 \\
\hline
\end{tabular}

The deflection responses of the fully-and partially-treated beams with and without the controller were evaluated in terms of transfer function (TF) of the tip displacement. Figure 7 compares the transfer function of tip displacement responses of the controlled beams with those of the corresponding passive beams $(B=0)$. The results show that the LQR control can reduce the deflection amplitudes corresponding to all the modes considered, irrespective of the MR fluid treatment. It should be noted that the TFs are presented upto only $500 \mathrm{~Hz}$, while the fifth mode of the partially treated beams lies at a frequency above 
$500 \mathrm{~Hz}$. The fully-and partially-treated beam (configuration B), in particular, yield most significant reductions in the tip deflections compared to those of the passive beams $(B=0)$. The configuration A of the partially-treated beam, however, exhibits relatively smaller reduction in the peak responses compared to the respective passive beam.

The most significant reductions are evident in deflection corresponding to the first mode for all the beams considered. For the fully-treated beam, the TF reveals that the LQR control yields $96 \%, 90 \%$, 93\%, 91\% and 99\% reductions in the peaks corresponds to the first five modes, respectively. It can also be seen that the first and fifth modes deflection peaks are entirely attenuated, as seen in Figure 7(a). The reductions in the peak deflections of the partially-treated beam (configuration B) corresponding to the first three modes are 99\%, 96\% and 98\%, respectively. The first and fourth modes deflections in this case are also entirely attenuated, as seen in Figure 7(c). The reductions in the peak amplitudes of partiallytreated beam, configuration A, are relatively much smaller than those observed for configuration B for all the modes, as seen in Figure 7 (b). This is mostly caused by the distribution of the MR fluid segments near the support, and is consistent with the free vibration responses presented in Figures 5 and 6.

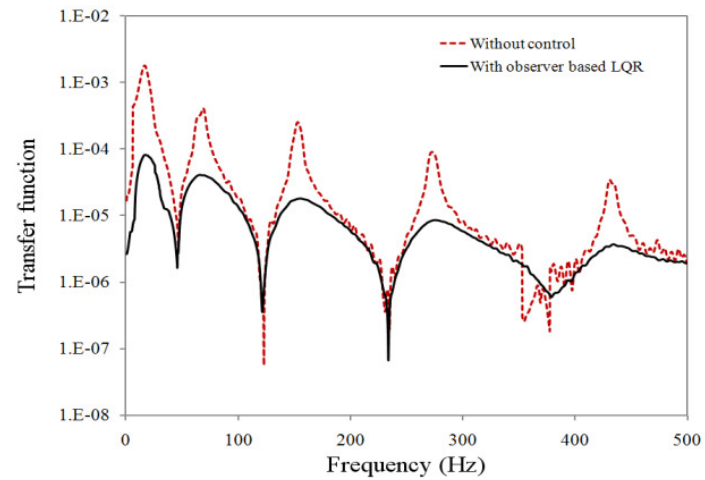

(a)

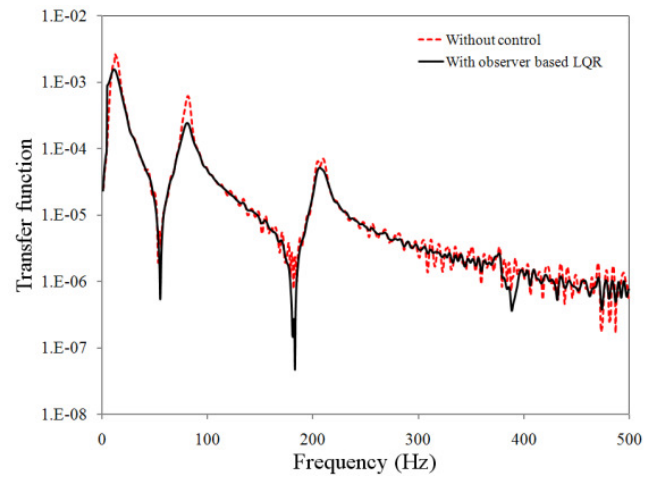

(b)

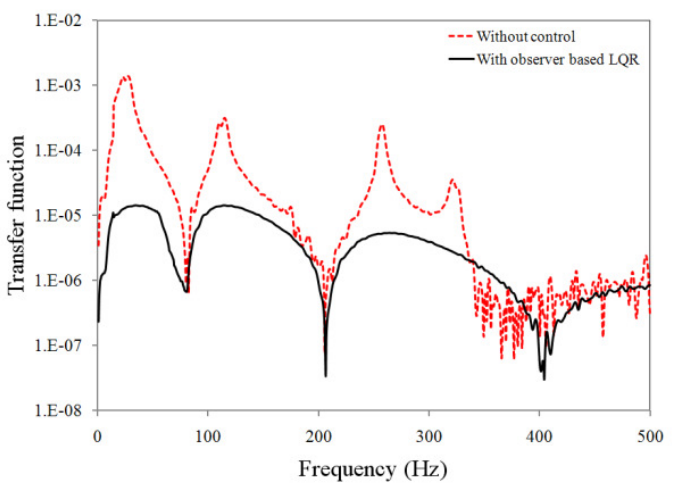

(c)

Figure 7. Comparisons of the transfer functions of the tip displacement responses of beams with and without observer based LQR control: (a) Fully treated beam; (b) configuration A; and (c) configuration B.

\subsection{Flexural mode shape (FMS) based LQR control}

Simulations are performed to examine the validity of the flexural mode shape (FMS) based optimal controller in estimating the state vector. The effectiveness of the FMS controller is subsequently evaluated in suppressing the free and forced vibration responses under both the impulse and white-noise force excitation at the tip. The effectiveness of the proposed FMS estimator and control method are demonstrated for the fully-treated beam alone, while the simulation parameters are taken as those described in previous section.

The validity of the FMS estimator is initially investigated considering the impulse excitation for the passive and controlled fully-treated beams. For this purpose, the finite element model was analyzed to 
yield the transverse deflection amplitudes obtained at $x=l=300 \mathrm{~mm}$ and $x=l / 2=150 \mathrm{~mm}$, which were applied to estimate the deflections at different coordinates, using (19). The estimated deflection responses at different locations were then compared with those derived from the FE model to examine the validity of the FMS estimator. The results revealed very good state estimation for both the passive and the controlled fully-treated beams. As an example, Figures 8(a) and 8(b) compare the estimated deflection responses of the passive beam with those derived from the finite element model at $x=100 \mathrm{~mm}$ and $x=$ $200 \mathrm{~mm}$, respectively. For the purpose of clarity, the responses are compared only in the 0 to $1 \mathrm{~s}$ interval, which invariably show that the estimated and computed responses overlap. Figures 9(a) and 9(b) present comparison of the computed and estimated deflection responses of the fully-treated beam employing the FMS-based LQR controller at $x=100 \mathrm{~mm}$ and $x=200 \mathrm{~mm}$ which further validate the FMS based estimator.

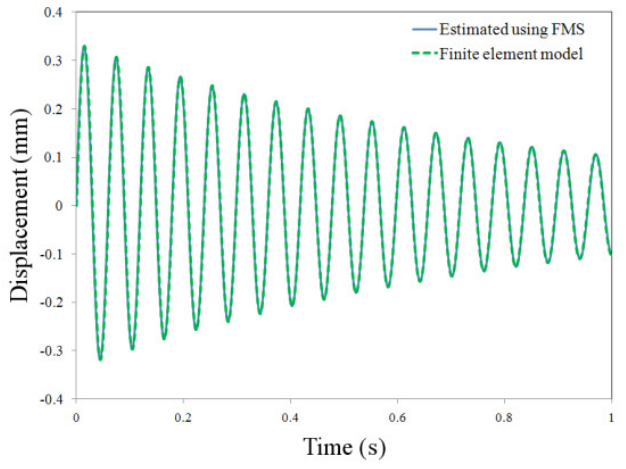

(a)

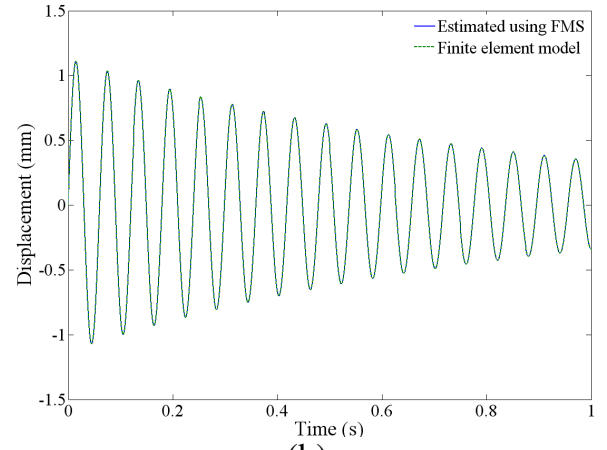

(b)

Figure 8. Comparisons of deflection responses of the fully treated passive beam estimated using FMS and the finite element model: (a) $x=100 \mathrm{~mm}$; and (b) $x=200 \mathrm{~mm}$

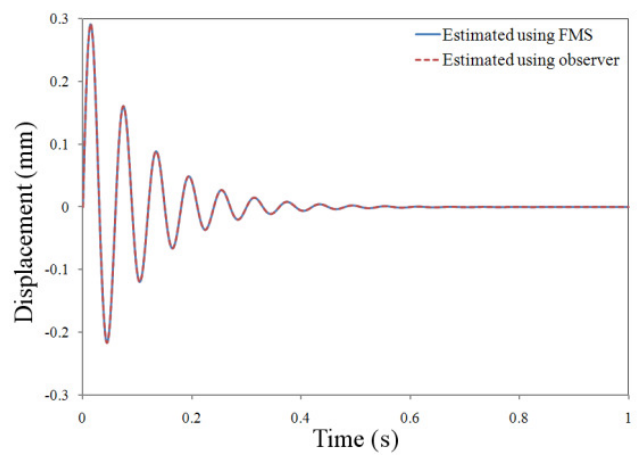

(a)

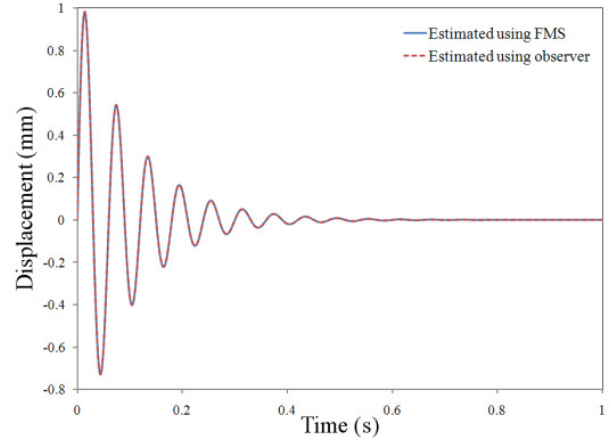

(b)

Figure 9. Comparisons of deflection responses of the fully treated beam estimated using FMS based and observer based LQR controllers : (a) $x=100 \mathrm{~mm}$; and (b) $x=200 \mathrm{~mm}$

From Figures 8 and 9, it is evident that the FMS-based LQR control can effectively adapt to the state of the beam. The proposed limited state control can thus yield vibration attenuation performance similar to the full state observer based control. Furthermore, the tip deflection responses of the fullytreated beam with and without FMS based LQR controller are shown in Figure 10 under the unit impulse excitation. The results show that the settling time and peak displacement has been significantly reduced. The settling time of the controlled beam is in the order of $0.59 \mathrm{~s}$, which is significantly lower than $4.325 \mathrm{~s}$ of the passive beam $(B=0)$. 


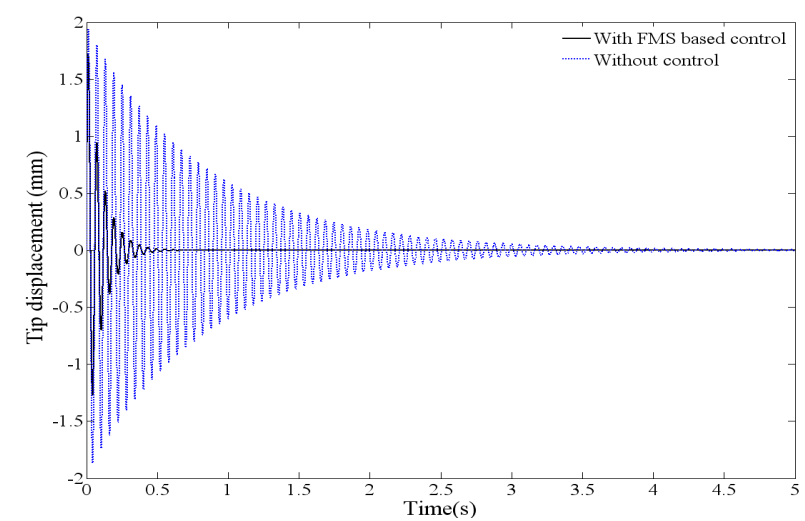

Figure 10. Open and closed loops responses at the tip of the fully treated MR sandwich beam under the impulse disturbance at the tip of the beam using FMS based controller.

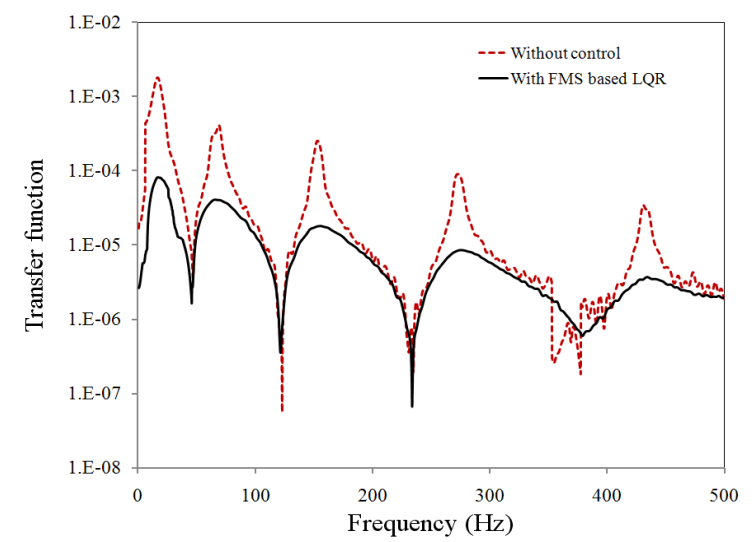

Figure 11. Comparisons of the transfer functions of the tip displacement responses of the fully treated beam with and without FMS based LQR control

The deflection responses of the fully treated beam with and without the FMS based control are further evaluated under a white-noise force excitation applied at the tip. Figure 11 compares the transfer function of tip displacement responses of the controlled beam with that of the corresponding passive beam $(B=0)$. The results show that the FMS based LQR control can reduce the deflection amplitudes corresponding to all the modes considered in the $0-500 \mathrm{~Hz}$ frequency range. The TF reveals that the FMS based LQR control yields the deflection reduction as good as the observer based LQR. The reductions in the peaks corresponds to the first five modes are $96 \%, 90 \%, 93 \%, 91 \%$ and $99 \%$, respectively. It can also be observed that the first and fifth modes deflection peaks are entirely attenuated. This confirms the effectiveness of the FMS-based limited state optimal controller.

\section{Conclusions}

In this study, the semi-active vibration control of a multilayer beam fully-and partially -treated with MR fluid has been analyzed. The results of the study show that the full-state observer based LQR control can yield substantial reductions in the settling time and free-and forced vibration responses of the beams treated either fully or partially with MR-fluid under an impulse excitation. The observer based LQR control of the fully and partially treated beams resulted nearly $85 \%$ reduction in the free-vibration settling time, and $25 \%$ reduction in the tip deflection when compared to that of the passive beams. The vibration responses to a white noise force excitation applied at the tip also revealed nearly $90 \%$ reductions in the deflections amplitudes corresponding to the higher modes for both the fully and partially-treated beams while the deflection corresponding to the first mode is entirely attenuated. The limited state control synthesis proposed on basis of the flexural mode shapes of the beam would also resulted in comparable vibration spectra, while reducing the number of feedback variables and the hardware implementation. The approach based on the known flexural mode shapes of the beam and measurements of only a few state variables provided effective estimates of the state vector. The controlled and uncontrolled deflection responses of the beam using FMS based control method evaluated under an impulse and a white-noise force applied at the tip of the beam confirms the effectiveness of the FMS-based limited state controller. The implementation of the controller, however, would require further developments in compact electromagnets to apply the controlled magnetic field on the surfaces of the treated structures.

\section{References:}

Ahn Y, Yang B Ahmadian M and Morishita S, 2005, A Small sized variable damping mount using magnetorheological fluid J. Intell. Mater. Sys. Struct., 16(2), 127-133.

Baillargeon B P and Vel S S, 2005, Active vibration suppression of sandwich beams using piezoelectric shear actuators: Experiments and numerical simulation J. of Intell. Mater. Sys. 
Struct., 16(6), 517-530.

Burl J B, 1999, Linear optimal control, Addison-Wesley, California.

Carlson J D and Weiss K D, 1994, A growing attraction to magnetic fluids, Mac. Desgn, 66 (15), 61-64.

Choi S B, 1999, Vibration control of flexible structures using ER dampers, J. of Dynamic Sys., Measurement and Contl., Transaction of ASME, 121(1), 134- 138.

Choi Y, Sprecher A F and Conrad H, 1990, Vibration characteristics of a composite beam containing an electrorheological fluid, J. Intell. Mater., Sys. and Struct. 1(1), 91-104.

Choi Y T, Wereley N M and Jeon Y S, 2005, Semi-active vibration isolation using magnetorheological isolators, J. of Aircraft, 42 (5), 1244-1251.

DiTaranto R A, 1965, Theory of vibratory bending of elastic and viscoelastic layered finite-length beams, J. of Appl. Mech., Transactions of ASME, Ser. E, 87(2): 881-886.

Dyke,S.J., Spencer, B.F. Sain, M.K. and Calrson, J.D. 1998, “An Experimental Study of MR Dampers on Seismic Protection", Smart Materials and Structures, 7(5): 693-703.

Gandhi M V, Thomson B S and Choi S B, 1989, A new generation of innovative ultraadvanced intelligent composite materials featuring electro-rhoelogical fluids: An experimental investigation", J. of Compo. Mater., 23 (12), 1232-1255.

Haiqing G, King L M and Cher T B, 1993, Influence of a locally applied electrorheological fluid layer on vibration of a simple cantilever beam", J. of Intell. Mater., Sys. and Struct., 4(3), 379384.

Haiqing G and King L M, 1997, Vibration characteristics of sandwich beams partially and fully treated with electrorheological fluid", J. of Intell. Mater. Sys. and Struct., 8(5), 401-413.

Harris C M, 1987, Shock and vibration handbook, McGraw-Hill, New York.

He H Q, Ng T Y, Sivashankar S. and Liew K M, 2001, Active control of FGM plates with integrated piezo-electric sensors and actuators," Int. J. of Solids and Struct., 38(9), 16411655.

Hu Q and Ma G, 2005, Variable structure control and active vibration suppression of flexible spacecraft during attitude maneuver, Aerospace Science and Tech., 9(4), 307-317.

Lam M J, Inman D J and Saunders W R, 1997, Vibration control through passive constrained layer damping and active control, J. of Intell. Mater. and Struct., 8(8), 663-677.

Lara-Prieto V N, Parkin R, Jackson M, Silberschmidt V and Kesy Z 2010, Vibration characteristics of MR cantilever sandwich beams: Experimental study, Smart Mater. and struct., 19(1), 015005 (9pp).

Lee U and Kim J, 2001, Spectral element modeling for the beams treated with active constrained layer damping, Intl. J. of Solids and Struct., 38, (32-33), 5679-5702.

Leitmann G and Reithmeier E, 1993, Semi active control of a vibrating system by means of electrorhelogical fluids, Dyn. and contl., 3(1), 7-33.

Leitmann G, 1994, Semi active control of vibration attenuation, J. of Intell. Mater. Sys. and Struct., 5(6): 841-846.

Liao W H and Wang, D H, 2003, Semi-active vibration control of train suspension systems via magnetorheological dampers, J. of Intell. Mater. Sys. and Struct. , 14 (161-172).

Liu Y, Water T P and Brennan M J, 2005, A comparison of semi-active damping control strategies for vibration isolation of harmonic disturbances", J. of Sound and Vib., 280(12), 21-39.

Mutambara A G O, 1999, Design and analysis of control systems, CRC press, Florida.

Nishitani A and Inoue Y, 2001, Overview of the application of active/semi-active control to building structures in Japan, Earthquake Eng. and Struct. Dynamics 30, 1565-1574.

Ogata K, 2008, MATLAB for control engineers, Pearson Prentice Hall, New Jersey.

Pranoto T, Nagaya K, and Hosoda A, 2004, Vibration suppression of plate using linear MR fluid passive damper, J. of Sound and Vib., 276 (3-5), 919-932.

Rajamohan V, Sedaghati R and Rakheja S 2010a, Vibration analysis of a multi-layer beam 
containing magnetorheological fluid, Smart Mater. and Struct., 19(1), 015013(12pp).

Rajamohan V, Rakheja S and Sedaghati R, 2010b, Vibration analysis of a partially treated multi- layer beam with magnetorheological fluid, J. of Sound and Vib., 329(17), 3451-3469.

Rajamohan V, Sedaghati R and Rakheja S, 2010c, Optimum design of a multilayer beam partially treated with magnetorheological fluid, Smart Mater. and Struct., 19(6), 065002 (15pp).

Sadri A M, Wright J R and Wynne R J, 1999, Modelling and optimal placement of piezoelectric actuators in isotropic plates using genetic algorithm, Smart Mater. and Struct., 8(4), 490-498.

See H, 2004, Advances in electro-rheological fluids: Materials, modeling and applications, J. of Indust. and Eng. Chemistry, 10(7), 1132-1145.

Sethi V and Song G, 2005, Optimal vibration control of a model frame structure using piezoelectric sensors and actuators, J. of Vib. and Contl, 11(5), 671-684.

Shaw J, 2000, Hybrid control of a cantilevered ER sandwich beam for vibration suppression, J. of Intell. Mater. Sys. and Struct., 11(1), 26-31.

Spencer B, Dyke S, Sain M and Carlson J, 1997, Phenomenological model of magnetorheological damper, ASCE J. of Eng. Mech., 123(3), 230-238.

Spencer Jr. B F and Nagarajaiah S, 2003, State of the art of structural control, J. of Struct. Eng., 129(7), 845-856.

Stanway R, Sproston J L and El Wahed A K, 1996, Applications of electrorheological fluids in vibration control: A survey, Smart Mater. and Struct., 5(4), 464-482.

Sun Q, Zhou J X and Zhang L, 2003, An adaptive beam model and dynamic characteristics of magnetorheological materials, J. of Sound and Vib., 261(3), 465-481.

Varadarajan S, Chandrashekhara, K and Agarwal S, 2000, LQG/LTR-based robust control of composite beams with piezoelectric devices, J. of Vib. and Cntrl, 6 (607-630).

Wang G and Wereley N M., 2002, Spectral finite element analysis of sandwich beams with passive constrained layer damping, J. of Vib. and Acoustics, 124(3), 376-386.

Yalcintas M and Coulter J P, 1995, Analytical modeling of electrorheological materials based adaptive beams, J. of Intell. Mater., Sys. and Struct., 6(4): 488-497.

Yalcintas M and Coulter J P, 1998, Electrorheological materials based non-homogeneous adaptive beams, Smart Mater. and Struct., 7(1), 128-143.

Yalcintas M and Dai H, 1999, Magnetorheological and electrorheological materials in adaptive structures and their performance comparison, Smart Mater. and Struct., 8(5), 560 573.

Yalcintas M and Dai H, 2004, Vibration suppression capabilities of magneto-rheological materials based adaptive structures, Smart Mater. and Struct., 13(1), 1-11.

Yao G Z, Yap F F, Chen G, Li W H and Yeo S H, 2002, MR damper and its application for semi-active control of vehicle suspension system, Mechatronics, 12(7), 963-973.

Yeh Z F and Shih Y S, 2006, Dynamic characteristics and dynamic instability of magnetorheological based adaptive beams", J. of composite Mater., 40(15), 1333-1359. 